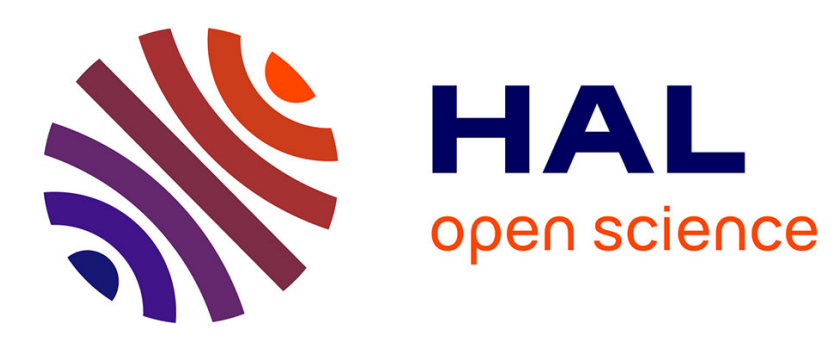

\title{
Efficient Placement of Light Splitters for Heterogeneous Multicast Traffic in Optical Networks
}

\author{
Shadi Jawhar, Bernard Cousin, Samer Lahoud
}

\section{To cite this version:}

Shadi Jawhar, Bernard Cousin, Samer Lahoud. Efficient Placement of Light Splitters for Heterogeneous Multicast Traffic in Optical Networks. High Capacity Optical Networks and Enabling Technologies (HONET), Dec 2010, Le Caire, Egypt. pp.204 - 209, 10.1109/HONET.2010.5715774 . hal01184103

\section{HAL Id: hal-01184103 \\ https://hal.science/hal-01184103}

Submitted on 12 Aug 2015

HAL is a multi-disciplinary open access archive for the deposit and dissemination of scientific research documents, whether they are published or not. The documents may come from teaching and research institutions in France or abroad, or from public or private research centers.
L'archive ouverte pluridisciplinaire HAL, est destinée au dépôt et à la diffusion de documents scientifiques de niveau recherche, publiés ou non, émanant des établissements d'enseignement et de recherche français ou étrangers, des laboratoires publics ou privés. 


\title{
Efficient Placement of Light Splitters for Heterogeneous Multicast Traffic in Optical Networks
}

\author{
Shadi Jawhar, Bernard Cousin, Samer Lahoud \\ IRISA laboratory, University of Rennes 1, Campus universitaire de Beaulieu, 35042, France \\ Bernard.Cousin@irisa.fr
}

\begin{abstract}
When multicasting in optical networks is implemented within the switching control plane, it combines the efficiency of multicast tree along with high speed and low delay of optical communications. Multicast nodes must be equipped with light splitters. Light splitters are expensive equipment. Therefore, a limited number of optical nodes will have this splitting capability. A good placement of optical splitters can increase the efficiency of the multicast signaling and routing techniques on one hand, and reduce the number of those splitters on the other hand. This leads to faster multicast trees setting up, lower data transmission delays, and less traffic on the network links; thus saving of optical links capacity for other multicast and unicast transmissions. In order to achieve efficient multicasting in optical network, we propose to take into account network characteristics (link capacity and node degree) when placing the optical splitters. The benefits of the smart placement of light splitters will be clearly shown in heterogeneous optical networks, where multicast traffic is not uniformly distributed over the network, and optical links connecting different nodes in the network have different characteristics.
\end{abstract}

\section{INTRODUCTION}

To assure the deployment of native optical multicasting, optical nodes need to be able to switch an incoming optical signal and copy it to more than one output interface; thus multicast nodes must be equipped with light splitters. Light splitters are expensive devices; in consequence it will be very expensive to implement splitters on all optical switches. Therefore, in an optical network, a limited number of optical nodes will have this splitting capability. Advanced studies [1] [2] show that at least $30 \%$ of the network nodes must be equipped with splitters in order to have a compromise between the multicast routing efficiency and the cost of the nodes with optical splitters. Al good placement of optical splitters can increase the efficiency of the multicast signaling and routing techniques on one hand, and reduce the number of those splitters (thus the cost of the network) on the other hand. This leads to faster multicast trees setting up, lower data transmission delays, and less traffic on the network links; thus saving of optical links capacity for other multicast and unicast transmissions.

In order to efficiently reuse usual multicast tree computation algorithms, and benefit from all the optical switching capability, light splitters must be placed where splitting is required. To generate multicast trees that benefits from all-optical switching [3] [4], optical nodes must be able to switch an input wavelength to more than one output.

We propose to define optical link weight based on their characteristics from one side, and on the multicast traffic provisioned and expected on the other side. Placement of the optical splitters based on these factors leads to more efficient tree generation when deploying multicast algorithms in optical networks.

In section two, a brief description of how multicasting is deployed over optical networks by constructing light trees to deliver data to group members. Complex and multiple light trees are built for a single group in networks where not all network nodes are multicast capable. In section three, relative work on how to place those splitters in the network is given. In section four, a new placement mechanism is proposed, it explains how to place those splitters taking into consideration new parameters that are based on provisioned multicast traffic and link characteristics of the network. In the last section, simulation is done, and results of the performance evaluation shows that efficient placement of those splitters based on links characteristics may enhance the multicast routing from one side, and reduce the numbers of splitters needed from the other side.

\section{Multicasting OVER Optical NetWorks}

Constructing multicast light trees in optical networks offers the ability to generate multiple light paths. Each path is a set of consecutive wavelength switching [5] occurring in the optical layer of intermediate nodes. Grouping several point to point light splitting paths LSPs can generate the trees spanning from the source to the group members. "All optical switches" [3] [4] reduces the time lost in the optical to electrical conversion and vice-versa.

An all-optical network is composed of OXCs. An Optical Cross Connect (OXC) is designed to switch an optical signal from an input port to an output port. Generally, the output signal uses the same wavelength as the input signal. Thus light paths are built, as they pass through one or more OXCs on which optical signal switching can be efficiently performed in the physical layer (without the need to go from the optical layer to the electrical layer and vice versa). 
An ordinary optical node is incapable of doing splitting in the optical layer. It can only convert the wavelength into an electrical signal, translate it into a data frame, duplicate the frame in memory and then send the multiple copies of the frame by translating them back in the optical domain. For the $\mathrm{OXC}$ to be able to do the multicasting in the optical layer, it must be equipped with an optical light splitter. For instance, Multicast-Capable optical cross connect MC-OXC [6] can be an optical switch that consists of a $\mathrm{P} \times \mathrm{P}$ splitter-and-delivery (SaD) switch [6], $\mathrm{P}$ de-multiplexers to extract individual inputs wavelengths and $\mathrm{P}$ multiplexers to combine the output wavelengths.

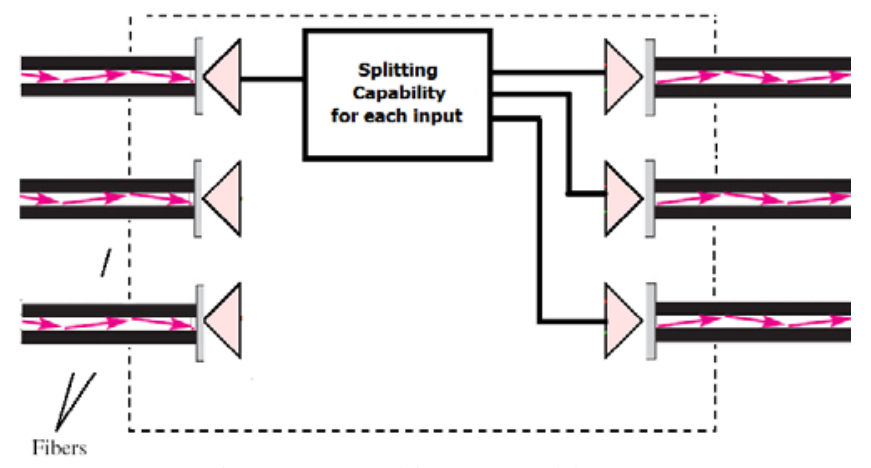

Figure 1 - Multicast Capable-OXC

The high cost of those splitters makes all-optical switches with multicast capability very expensive. Previous work [1] [2] shows that placing splitters in $30 \%$ of the optical nodes is sufficient to generate one optical tree for each group. The optical tree connects the source to each member of the group without conversion to the electrical domain. This work is based on networks where multicast traffic is assumed uniformly distributed over the network, without taking into consideration that in most of the network, multicast traffic is heterogeneous.

\section{RELATED WORK}

Relevant propositions for generating multicast trees on optical networks in which not all nodes have light splitters have been presented in [1][7][8] and [9]. Some of these proposals are based on additional signaling performed during or before data forwarding to generate trees, taking into consideration splitters location. Others use re-route-to-source asking the source to send a second stream of data to be able to do multicasting in the optical layers. It starts by generating one multicast tree to span all the destination nodes, and then it detects multicasting nodes that has no splitting capability, and resolves the issue by reroute to source technique. Other propositions generate multiple trees [7] in order to resolve the lack of splitting capability. The efficiency of those algorithms will depend on the number and location of splitters.

Moreover, some papers determine the number of MC-OXCs that correspond to an acceptable cost and good performing. The recommended percentage of nodes possessing light splitters and branching capability is 25 to $30 \%$ [1] [2]. Moreover additional work has been done to distribute light splitters and wavelength converters in a way to minimize the overall number of wavelength channels required by any multicast traffic on a given network topology. In [10], the authors propose to select the node to be equipped by a splitter based on the node degree. The degree of a node is the number of adjacent nodes with direct links connected to it.

The latter proposition, which is based on node degree has some drawbacks. First, the authors make the assumption that the multicast traffic is homogeneously spread all over the network nodes. It is not always the case; For instance, some network nodes may mainly host data servers whereas other nodes may mainly host user terminals, or some nodes in the network may heavily use videoconference applications and exchange multicast traffic whereas other may not. Second, the authors of the above proposition make the assumption that all the network links have the same capacity, and it is obviously not true in real networks. These assumptions induce inefficient splitter placement, which leads to inefficient trees and spoiling of network resources. Therefore, in order to achieve efficient multicasting in optical network, network characteristics have to be taken into account when placing the optical splitters. In the next section, we show how to take into consideration these characteristics.

\section{Placement OF Light SPlitters}

Given a network topology made up of optical nodes interconnected by optical links. In order to have a good distribution of splitters over the network, different parameters must be taken into account. As seen in previous work, node degree (number of neighbors) is one of these parameters. The provisioned multicast traffic is a factor that allows placing splitters in locations that will be the most useful once multicast trees are being generated. It is very important to place splitters where multicasting will occur more frequently.

When a node must have several downstream nodes and does not possess light splitting capability, then several trees [7] have to be created. In this case, the link usage will increase (for instance the same link could have to support several copies of the same signal on different wavelengths) and the multicast structure generated (composed by several trees) will be less efficient. As a result, efficient placement of splitters will increase the efficiency of the generated trees.

Optical links capacity needs also to be taken into account. Each link in the network has its own capacity which determines the amount of flows it can carry simultaneously. The more traffic is being transmitted on a link, the less residual capacity is available for other transmissions. The capacity of links is mostly determined during network design, by the traffic requirements. In consequence, high capacity must be assigned to links where high traffic is expected.

Generally, each optical link in the network is given a specific weight or cost. We assume that the cost of an optical 
link is determined in terms of the link capacity. This is cost is inversely proportional to the capacity of the link. We assume that not all links in the network are identical and that each link has its own capacity, thus the splitter placement can no more be based on the number of links connected to each node (the node degree).

On contrast, the splitter placement must be based on the number of links on one side, and the cost of each link on other side. To combine these two objectives, we introduce the concept of weighted nodal degree. We consider the network topology shown in Figure 2. This is a well known and wellconnected carrier's backbone topology. Assuming a network of 24 nodes, let suppose that a total of 6 splitters has to be placed. Distributing these splitters on the nodes with the highest nodal degrees (as in Node Degree Splitter Placement, NDSP) [10] means that they must be placed on nodes $6,7,9$, 11, 16, and 17. Each of those nodes has direct links with 5 adjacent nodes.

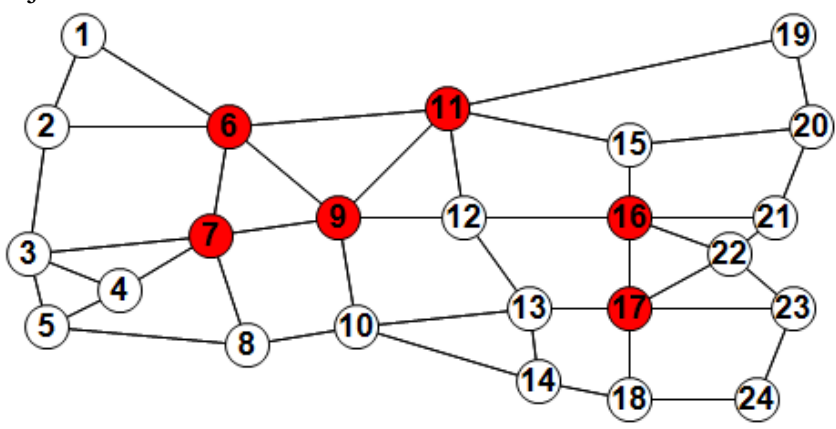

Figure 2 - Splitters distributed based on nodes degree

\begin{tabular}{|c|c|}
\hline \multicolumn{2}{|c|}{ Table 1 : Nodal Degrees } \\
\hline Node & Node degree \\
\hline $\mathbf{6 , 7 , 9 , 1 1 , 1 6 , 1 7}$ & $\mathbf{5}$ \\
\hline $3,10,12,13,22$ & 4 \\
\hline $2,4,5,8,14,15,18,20,21,23$ & 3 \\
\hline $1,19,24$ & 2 \\
\hline
\end{tabular}

The node-degree method of placing splitters in the network does not take into consideration any of the optical links characteristics. This method is simple: the data needed to perform the splitter placement with this method is easy to obtain. However all links are considered the same without paying attention on link capacity or wavelength availability. In consequence the splitter placement may turn out to be inefficient, (in accordance with multicast traffic requirement).

In order to place splitters efficiently, each optical link in the network is assigned a weight factor which defines the cost to use the link. Based on multicast traffic provisioning, optical links are designed each with a capacity corresponding to the flow expected to be transmitted over this link.

Figure 3 shows the same network topology as Figure 2 for which each optical link is assigned a specific weight. We assume that this weight is in fact based on the link capacity.
Weighted node degrees are computed as the sum of costs of all links attached to the node. We can see that the weighted node degrees (see Table 2) are different from strict node degrees (see Table 1).

For example, node 10 is connected to four adjacent nodes, but the cost of those four links is high. As a result, this node has a high weighted nodal degree when calculated based on the cost of the four links. The WNDSP of a node is the sum of the links weights connected to it, which is equal to 10 for node 10.

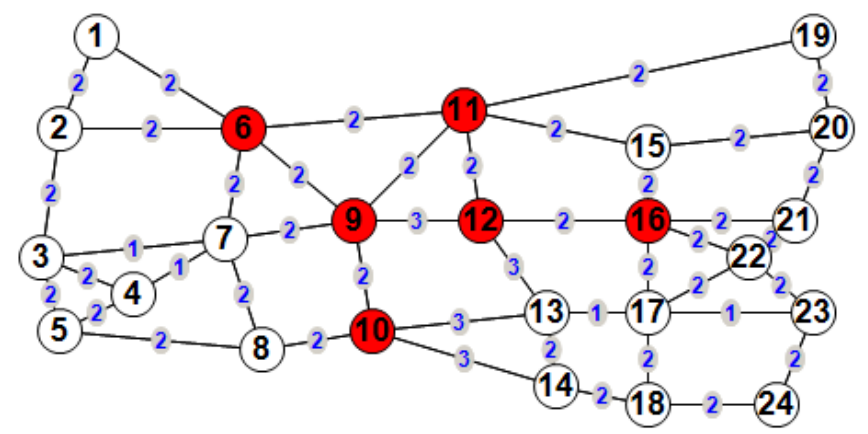

Figure 3 - Splitters distributed based on links cost

\begin{tabular}{|c|c|}
\hline \multicolumn{2}{|c|}{ Table 2: Weighted nodal degrees } \\
\hline Node & Weighted Node Degree \\
\hline $\mathbf{9}$ & $\mathbf{1 1}$ \\
\hline $\mathbf{6 , 1 0 , 1 1 , 1 2 , \mathbf { 1 6 }}$ & $\mathbf{1 0}$ \\
\hline 13 & 9 \\
\hline $7,17,22$ & 8 \\
\hline 3 & 7 \\
\hline $2,8,14,15,18,20,21$ & 6 \\
\hline 4,23 & 5 \\
\hline $1,5,19,24$ & 4 \\
\hline
\end{tabular}

Based on the Weighted Node Degree Splitter Placement WNDSP, the five splitters are now placed on nodes $6,9,10$, 11,12 , and 16 . The two splitters that were previously placed on nodes 7 and 17 are now relocated to nodes 10 and 12 . Nodes 10 and 12 will benefit more from the splitters because of several reasons. This is because the use of links attached to those nodes will cost a higher loss because those links are higher in capacity (thus in weight).

The cost of excessive use of those links will be higher when deploying any of the assumed propositions to solve the problem of incapability of multicasting in the optical layer. Whether signaling or data traffic in case multiple trees generated or rerouting to source happened, this cost shows high negative effect. An example of this is link 10-13 or link 12-13, because their weight is high and thus transmission on these is not recommended. Another reason is that more multicast traffic is expected on the links attached to this node and this is reflected by their assigned weights. 


\section{Simulations AND RESUltS}

In order to demonstrate that the link parameters play an important role in the splitter placement, simulations are preformed to compare the splitter placement based on the node degree (NDSP) and the other based on the weighted node degree (WNDSP).

\section{a. Cost of trees and beneficial use of splitters}

Considering the 24-nodes network topology described in the previous section, we first place splitters based on the number of links connected to each node. We consider 24 random multicast groups; for each multicast group, the source is placed on one different node. Group members are randomly chosen amongst the network nodes according to a uniform distribution law based on a given parameter: the number of group members. For each group, one shortest path tree is computed. We use groups of 3, 4, 5, 6 and 7 members to be able to simulate different sizes of groups. As a result, varying the source on all nodes of the network and randomly choosing members and group sizes will give more reliable simulation results.

Multicast trees will be able to perform all optical switching [11] [12] when the splitters are located on the nodes that are branching nodes for the shortest path tree. This demonstrates the idea that efficient placement of light splitters affects the generated trees: that is the efficiency of the tree generating algorithm.

In order to be able to evaluate our proposition, we propose to generate the multicast trees taking into account the splitter location. We calculate the tree cost in terms of number of links used for every multicast session, and in terms of the total cost of links used.

Table 3 shows the total count of links used in the trees generated for each multicast session. This represents the wavelength usage in the optical network. For instance for the groups of 3 members, the overall number of the links for the 24 multicast groups generated is 342 links. That makes in average 342/24 (i.e. 14.25) links used by each 3_member_group tree. As shown below, the number of links used in the generated trees shows an enhancement of about $4 \%$ when splitters are located according to our proposal.

\begin{tabular}{|c|c|c|c|}
\hline \multicolumn{3}{|c|}{ Table 3: Overall number of links used } \\
\hline \multirow{2}{*}{ Groups of } & NDSP & WNSDP & Improvement \\
& & & \\
\hline 3 Members & 342 & 328 & $4.3 \%$ \\
\hline 4 Members & 423 & 407 & $3.9 \%$ \\
\hline 5 Members & 501 & 478 & $4.8 \%$ \\
\hline 6 Members & 556 & 530 & $4.9 \%$ \\
\hline 7 Members & 606 & 592 & $2.4 \%$ \\
\hline
\end{tabular}

Figure 4 shows graphically the improvement in tems of number of links used to generate the multicast tree.

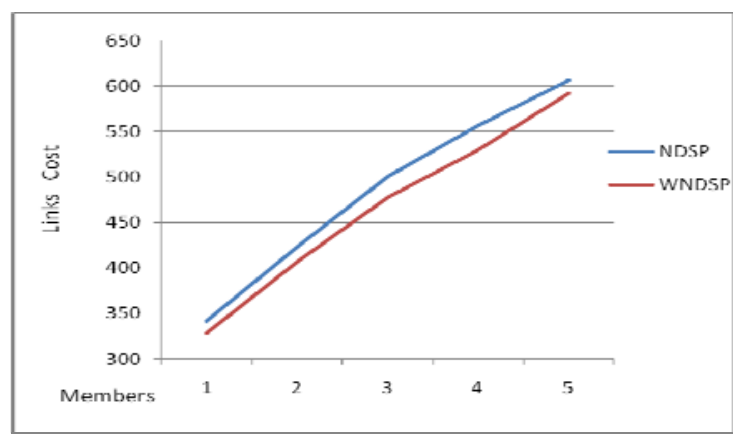

Figure 4 Improvement in overall number of links versus group size

Calculating the cost of multicast trees (with the link cost based on link weight) will give a more accurate evaluation of network resources used by the multicast trees. Indeed, we suppose that the more the capacity of a link is, the more the link by the multicast traffic will be used. This assumption is very realistic since during the network design phase, the capacity of a link is determined by the traffic expected on that link. As a result, Table 4 shows the total weighted cost of trees generated in terms of the sum of the weight of the links used in the generated trees. Here an enhancement of 5.5\% is shown once splitters are efficiently placed in the network according to WNDSP.

\begin{tabular}{|c|c|c|c|}
\hline \multicolumn{4}{|c|}{ Table 4: Total cost of links used } \\
\hline Groups of & NDSP & WNDSP & Improvement \\
\hline 3 Members & 700 & 664 & $5.4 \%$ \\
\hline 4 Members & 871 & 830 & $5.2 \%$ \\
\hline 5 Members & 1033 & 976 & $5.9 \%$ \\
\hline 6 Members & 1146 & 1082 & $5.9 \%$ \\
\hline 7 Members & 1243 & 1204 & $3.2 \%$ \\
\hline
\end{tabular}

Figure 5 shows graphically the improvement in terms of cost of links used, over all the multicast trees.

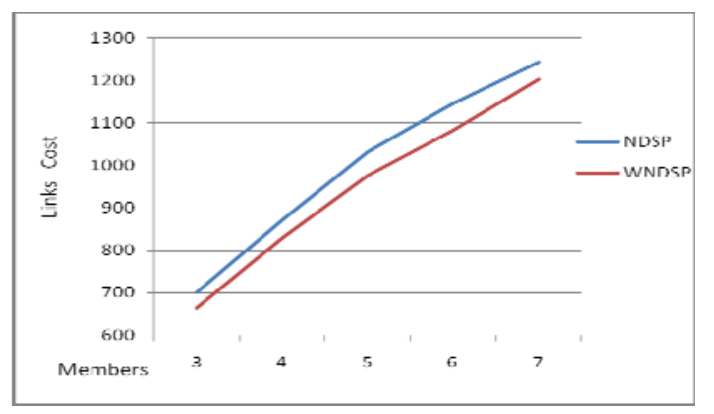

Figure 5 Improvement in total cost of links versus group size

b. Performance evaluation based on the provisioned multicast traffic 
In order to accurately define the link weights, simulating the provisioned multicast traffic and assigning link capacity based on the expected multicast traffic is a good factor to show the importance of placing splitters in the best locations. We consider a new network of 24 nodes divided into two parts. We assume that multicast traffic on one of the parts is 3 times more than the other. As a result link weights in one side are three times the other.

As shown in Figure 6, the weights of network links in the left part are three times those on the right side. Since the efficient placement of splitters depends on the weights of links connected to each network node, then with our algorithm the density of splitters that are placed in Part 2 is higher than in Part 1.

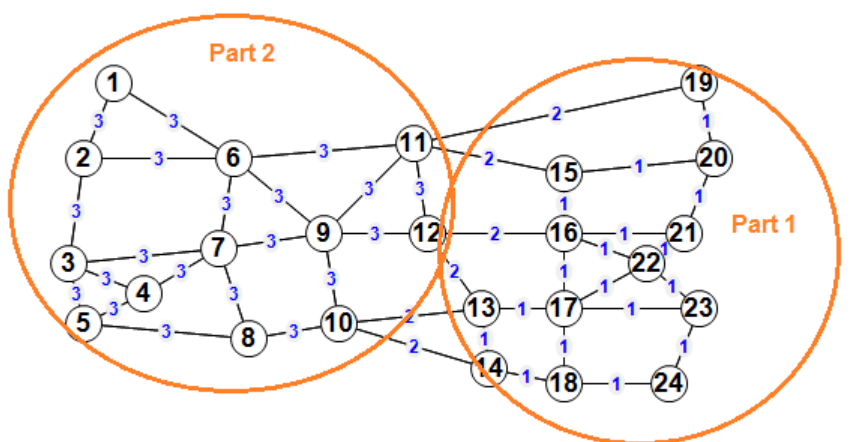

Figure 6 Links weights with multicast traffic provisioning.

Splitter placement with NDSP algorithm (resp. WNDSP algorithm) is shown in Figure 7 (resp. Figure 8).

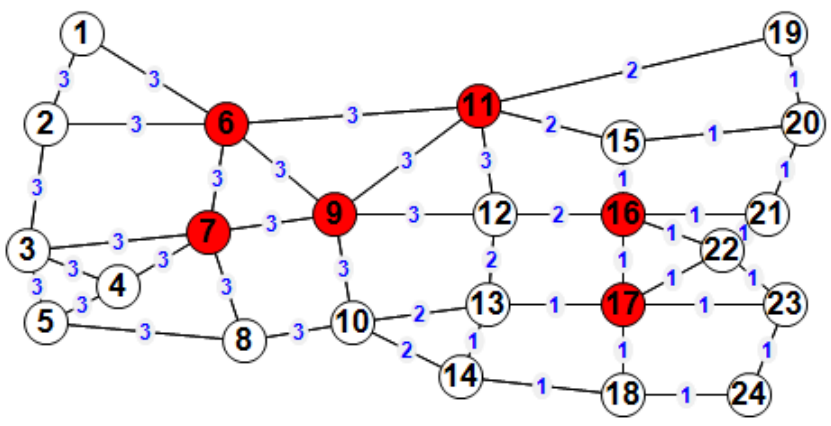

Figure 7 Splitters placement with NDSP

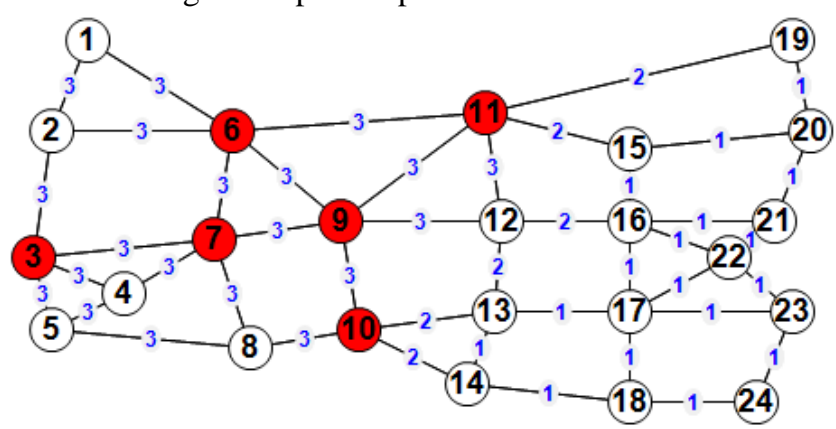

Figure 8 Splitters placement with WNDSP
Performance evaluations are done with six network nodes with splitting capability (i.e. $25 \%$ of nodes in the network have splitters), then seven (i.e. 29\% of nodes have splitters) and finally eight (i.e. $34 \%$ of nodes have splitters). We generate a number of multicast groups equal to the number of nodes, each time a different node is the group source. We assume that the multicast members are three times more often in Part 2 of the network than in Part 1, and we compare the cost of the generated trees in terms of overall number of links and total weighted cost of links.

\begin{tabular}{|c|c|c|c|}
\hline \multicolumn{4}{|c|}{ Table 5: 6 splitters placed } \\
\hline & NDSP & WNDSP & Enhancement \\
\hline $\begin{array}{c}\text { Overall number } \\
\text { of links used }\end{array}$ & 312 & 310 & $0.6 \%$ \\
\hline $\begin{array}{c}\text { Total cost of } \\
\text { links used }\end{array}$ & 730 & 720 & $1.38 \%$ \\
\hline $\begin{array}{c}\text { Branching } \\
\text { nodes with no } \\
\text { splitter }\end{array}$ & 320 & 307 & $4 \%$ \\
\hline
\end{tabular}

\begin{tabular}{|c|c|c|c|}
\hline \multicolumn{4}{|c|}{ Table 6: 7 splitters placed } \\
\hline & NDSP & WNDSP & Enhancement \\
\hline $\begin{array}{c}\text { Overall number } \\
\text { of links used }\end{array}$ & 312 & 310 & $0.6 \%$ \\
\hline $\begin{array}{c}\text { Total cost of } \\
\text { links used }\end{array}$ & 731 & 719 & $1.67 \%$ \\
\hline $\begin{array}{c}\text { Branching } \\
\text { nodes with no } \\
\text { splitter }\end{array}$ & 310 & 297 & $4.3 \%$ \\
\hline
\end{tabular}

\begin{tabular}{|c|c|c|c|}
\hline \multicolumn{3}{|c|}{ Table 7: 8 splitters placed } \\
\hline & NDSP & WNDSP & Enhancement \\
\hline $\begin{array}{c}\text { Overall number } \\
\text { of links used }\end{array}$ & 310 & 308 & $0.7 \%$ \\
\hline $\begin{array}{c}\text { Total cost of } \\
\text { links used }\end{array}$ & 728 & 714 & $1.93 \%$ \\
\hline $\begin{array}{c}\text { Branching } \\
\text { nodes with no } \\
\text { splitter }\end{array}$ & 284 & 276 & $2.9 \%$ \\
\hline
\end{tabular}

As shown in the tables above, the WNDSP algorithm results in a reduction in the overall number of links used to construct the tree on one side, and the total cost of links of the generated trees on the other side. 


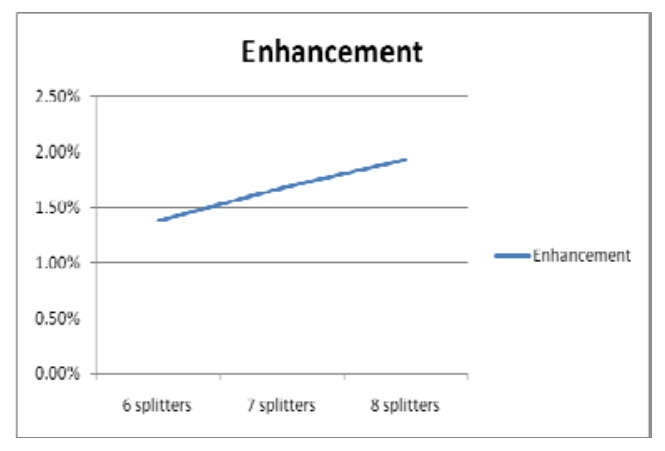

Figure 9 Enhancement versus total of cost of trees

For instance, with 7 splitters there are 284 branching nodes in the 24 trees computed by the shortest path tree which has no splitter if we use the NDSP algorithm. The best result is obtained when those splitters are distributed based on the WNDSP algorithm. This resulted with approximately 3\% enhancement.

Moreover, placement of 6 splitters provided by the WNDSP algorithm can give better results than the placement of 7 splitters by NDSP algorithm. Thus, WNDSP algorithm can also reduce the total number of splitters needed, and as a result the total cost of the network.

\section{CONCLUSION}

This work studies an important parameter for the deployment of multicasting over optical networks: placement of light splitters based on optical link capacity. In case of heterogeneous distribution of multicast traffic, the links connecting nodes in the network have different capacities. This difference in the links capacities will affect the way splitters are distributed in the network. Efficient placement of light splitters leads to efficient trees generated, that benefit from the multicast reduce of traffic, the optical links speed and performance, and finally the ability to do the data forwarding all in the optical layer.

In order to be able to use the same multicast algorithms and protocols achieved over IP networks and deploy those over optical networks, all optical cross connects must be equipped with light splitters. Due to the high cost of having splitters on all optical nodes in the network, a limited number of the nodes are chosen to be equipped to splitters. Therefore, usual multicast algorithms and protocols cannot be used.

Due to lack of splitters on all nodes in the network, specific signaling protocols, multicast traffic rerouting, or multiple tree generations must be used in order to generate the multicast traffic from the source to the destinations. Each of those will add some cost to the generated trees in terms of QoS, traffic, duplicates, delay or construction time.
In order to reduce this additional cost, splitters must be distributed in an efficient way taking into consideration the network topology, relative positions of optical nodes links, and the physical characteristics of the optical links. This also leads to reduce in the delay of delivering data to the group members.

\section{REFERENCES}

[1] Maher Ali, Jitender Deogun. "Allocation of Splitting Nodes in All-Optical Wavelength-Routed Networks”, Photonic Network Communications, 2000.

[2] Shuguang Yan, Jitender Deogun, Maher Ali. "Routing in sparse splitting optical networks with multicast traffic", Computer Networks, 2002

[3] W. S. Hu, Q. J .Zeng. "Multicasting Optical Cross Connects Employing Splitter-And-Delivery Switch", IEEE Photonics Technology Letters, 1998.

[4] Chunming Qiao, Myoungki Jeong, Amit Guha, Xijun Zhang, John Wei. "WDM Multicasting in IP over WDM Networks", Seventh Annual International Conference on Network Protocols, 1999.

[5] Jianping Wang, Xiangtong Qi, Biao Chen. "Wavelength assignment for multicast in all-optical WDM networks with splitting constraints”, IEEE/ACM Transactions on Networking, 2006.

[6] Marcos Rogério Salvador, Sonia M. Heemstra de Groot, Diptish Dey. "An All-Optical WDM Packet-Switched Network Architecture with Support for Group Communication”, IEEE International Conference on Networking, 2001.

[7] Towsif Mannan, BinWang. "Dynamic multicast session provisioning in WDM optical networks with sparse splitting capability”, International Conference on Communications and Computer Networks, 2006.

[8] Fen Zhou, Miklos Molnar, Bernard Cousin. "Is Light-Tree Structure Optimal for Multicast Routing in Sparse Splitting WDM Networks?”, IEEE International Conference on Computer Communications and Networks (ICCCN), 2009.

[9] Fen Zhou, Miklos Molnar, Bernard Cousin. "Avoidance of Multicast Incapable Branching Nodes for Multicast Routing in WDM Networks”, Photonic Network Communications, 2009.

[10] Oliver Yu and Yuan Ca. "Placement of Light Splitters and Wavelength Converters for Efficient Multicast in All-Optical WDM Networks", IEICE Transactions on Information and Systems, 2006.

[11] Yufeng Xin, George N. Rouskas. "Multicast Routing Under Optical Layer Constraints”, IEEE Infocom, 2004.

[12] W.-Y. Tseng, S.-Y Kuo. "All-optical multicasting on wavelength routed WDM networks with partial replication”. IEEE, International Conference on Intelligence in Networks, 2001.

[13] R. Braudes and S. Zabele. "Requirements for Multicast Protocols”, IETF RFC 1458, 1993.

[14] D. Brungard. "Requirements for Generalized MultiProtocol Label Switching Routing for the Automatically Switched Optical Network”, IETF RFC 4258, 2005.

[15] D. Papadimitriou, J. Drake, J. Ash, A. Farrel, L. Ong. "Requirements for Generalized MPLS Signaling Usage and Extensions for Automatically Switched Optical Network”, IETF RFC 4139, 2005.

[16] B. Rajagopalan et al. "IP over Optical Networks: Architectural Aspects", IEEE Communications Magazine, 2000. 